\title{
Oxidant-NO dependent gene regulation in dogs with type I diabetes: impact on cardiac function and metabolism
}

\author{
Caroline Ojaimi*, Shintaro Kinugawa, Fabio A Recchia, Thomas H Hintze
}

\begin{abstract}
Background: The mechanisms responsible for the cardiovascular mortality in type I diabetes (DM) have not been defined completely. We have shown in conscious dogs with DM that: 1) baseline coronary blood flow (CBF) was significantly decreased, 2) endothelium-dependent (ACh) coronary vasodilation was impaired, and 3) reflex cholinergic NO-dependent coronary vasodilation was selectively depressed. The most likely mechanism responsible for the depressed reflex cholinergic NO-dependent coronary vasodilation was the decreased bioactivity of NO from the vascular endothelium. The goal of this study was to investigate changes in cardiac gene expression in a canine model of alloxan-induced type 1 diabetes.

Methods: Mongrel dogs were chronically instrumented and the dogs were divided into two groups: one normal and the other diabetic. In the diabetic group, the dogs were injected with alloxan monohydrate (40-60 mg/kg iv) over $1 \mathrm{~min}$. The global changes in cardiac gene expression in dogs with alloxan-induced diabetes were studied using Affymetrix Canine Array. Cardiac RNA was extracted from the control and DM $(n=4)$.

Results: The array data revealed that 797 genes were differentially expressed $(P<0.01$; fold change of at least \pm 2 ). 150 genes were expressed at significantly greater levels in diabetic dogs and 647 were significantly reduced. There was no change in eNOS mRNA. There was up regulation of some components of the NADPH oxidase subunits (gp91 by 2.2 fold, $P<0.03$ ), and down-regulation of SOD1 ( 3 fold, $P<0.001$ ) and decrease ( 4 - 40 fold) in a large number of genes encoding mitochondrial enzymes. In addition, there was down-regulation of $\mathrm{Ca}^{2+}$ cycling genes (ryanodine receptor; SERCA2 Calcium ATPase), structural proteins (actin alpha). Of particular interests are genes involved in glutathione metabolism (glutathione peroxidase 1, glutathione reductase and glutathione S-transferase), which were markedly down regulated.
\end{abstract}

Conclusion: our findings suggest that type I diabetes might have a direct effect on the heart by impairing NO bioavailability through oxidative stress and perhaps lipid peroxidases.

\section{Background}

Diabetes mellitus (DM) is a multifactorial disease which is characterized by hyperglycemia, lipoprotein abnormalities [1] and altered intermediary metabolism of major food substrates [2]. In addition, the generation of free radicals often worsens the complications of DM such as hypertension, atherosclerosis and microcirculatory disorders $[3,4]$. Changes in lipid levels and consequent disorders of lipid metabolism and stress have been observed

\footnotetext{
* Correspondence: caroline_ojaimi@nymc.edu

Department of Physiology, New York Medical College, Valhalla, NY 10595, USA
}

C 2010 Ojaimi et al; licensee BioMed Central Ltd. This is an Open Access article distributed under the terms of the Creative Commons Attribution License (http://creativecommons.org/licenses/by/2.0), which permits unrestricted use, distribution, and reproduction in any medium, provided the original work is properly cited. in DM [5]. Due to altered intermediary metabolism in $\mathrm{DM}$, a feature present in both type 1 and type $2 \mathrm{DM}$, patients with type $2 \mathrm{DM}$, even in the absence of clinical cardiovascular disease, have a reduced maximal oxygen consumption compared with non-diabetic people [6]. On the other hand, several studies have reported that the basal metabolic rate is increased in diabetic states $[7,8]$. This increase is a reflection of the increased metabolic demands in DM.

Diabetes mellitus is usually associated with coronary artery disease. Since the discovery of nitric oxide (NO), a number of studies have revealed impaired endothelium-dependent vasorelaxation in diabetic animals 
$[9,10]$. Our previous study [11] has indicated that reflex, $\mathrm{NO}$-dependent coronary vasodilation is depressed in conscious dogs after the development of alloxan-induced diabetes, suggesting a reduced ability of coronary blood vessels to produce NO. Furthermore, in another study [12], we have shown that: 1) kallikrein causes a decrease in $\mathrm{O}_{2}$ consumption in cardiac muscle from normal dogs, which is mediated by kinin and NO mechanisms; and 2) the modulation of $\mathrm{O}_{2}$ consumption by endogenous $\mathrm{NO}$ is depressed in cardiac muscle from diabetic dogs, whereas the modulation of $\mathrm{O}_{2}$ consumption by exogenous NO is still preserved. The mechanism responsible for the depressed modulation of $\mathrm{O}_{2}$ consumption by endogenous NO is likely because of the decreased release of NO from the vascular endothelium rather than mitochondrial dysfunction. Various studies have shown that there is also evidence for mitochondrial dysfunction in the heart from the diabetic rat and mouse [13-16]. In addition, another study by Linke et al [17] showed significant changes in the myocardial substrate (glucose) utilization in dogs only in the late stage of diabetes at a time when myocardial NO production is already decreased. DNA microarrays have been used in every aspect of biological and medical research, including obesity and type 2 diabetes mellitus (T2D) in human and various small animal models [18]. Therefore, we sought to investigate cardiac gene expression changes in a canine model of alloxan-induced type 1 diabetes. We subsequently, compared and contrasted these changes to the cardiac functional and metabolic effects that we previously observed.

\section{Methods}

\section{Surgical instrumentation and in vivo protocol}

The protocols were approved by the Institutional Animal Care and Use Committee of New York Medical College and conform to the guiding principles for the use and care of laboratory animals of the National Institutes of Health and the American Physiological Society. The loss of body weight in the diabetic dogs was specifically discussed at the meeting of the Institutional Animal Care and Use Committee. Dogs were weighed every other day.

\section{Surgical Preparation}

Male Mongrel dogs (24.5-29 kg body wt, $n=8$ ) were premedicated with acepromazine $(0.3 \mathrm{mg} / \mathrm{kg} \mathrm{im})$ and anesthetized with pentobarbital sodium ( $25 \mathrm{mg} / \mathrm{kg}$ iv). A thoracotomy was performed, and instruments were implanted for measurement of pressure, flow, and blood sampling as described previously [11].

\section{Induction of Diabetes in Conscious Dogs}

The protocol used for these groups of control and diabetic dogs was the same as reported in our previous studies [11,12,17]. Mongrel dogs were chronically instrumented for measurements of systemic hemodynamics. The control hemodynamics were recorded 10-14 days after the surgery. After the control recording, the dogs were divided into two groups: one normal $(n=4)$ and the other diabetic $(n=4)$. In the diabetic group, the dogs were injected with alloxan monohydrate (40-60 mg/kg iv) over $1 \mathrm{~min}$. Alloxan was prepared as a $5 \%$ solution in citrate buffer ( $\mathrm{pH} 4-4.5)$. Blood gases and plasma glucose were measured on days 3 and 7 after alloxan injection. In some dogs whose plasma glucose levels were below $200 \mathrm{mg} / \mathrm{dl}$ at day 3 after injection of alloxan, a second injection of alloxan was given. Only dogs with blood glucose $>200 \mathrm{mg} / \mathrm{dl}$ (fasted for at least $16 \mathrm{~h}$ ) on day 7 were included in the diabetic group. Before and after the development of diabetes, the dogs had free access to water. Systemic hemodynamics were measured again after 3-5 wk. For microarray analysis, DM dogs were sacrificed at 4 weeks after alloxan injection and the LV of the heart was harvested and stored at $-80^{\circ} \mathrm{C}$ for only two weeks before RNA isolation was performed and sent to microarray facility for quality control, labeling and hybridization to Affymetric microarray.

\section{Recording Techniques}

Arterial pressure, LV pressure, left circumflex coronary blood flow (CBF), and internal diameters were measured; heart rate (HR), mean arterial pressure, and mean CBF were derived in conscious dogs, as we described previously [11]. Measurements were recorded continuously throughout the course of the experiment. Baseline measurements were taken after the dog was resting quietly on the table for $40 \mathrm{~min}$.

\section{RNA isolation}

Total cardiac RNA was extracted at the same time from left ventricular tissue of control $(\mathrm{n}=4)$, and DM dogs $(\mathrm{n}=4)$ with a commercial RNA isolation kit using Trizol (TRI REAGENT, Sigma, Saint Louis Missouri) as described previously $[19,20]$. RNA samples were stored at $-80^{\circ} \mathrm{C}$ for two weeks before microarray labeling and hybridization. Two quality control measures are carried out on a small aliquot of the isolated RNA samples: 1) a spectrophotometric analysis to confirm the concentration and to detect contaminating proteins and other molecules, and 2) a size fractionation procedure using the Agilent Bioanalyzer 2100 (Agilent Technologies) to determine whether the RNA is intact. The later was performed at the Bionomics Research and Technology Center (BRTC), Rutgers University.

\section{Microarray labeling and hybridization}

The microarray labeling and hybridization procedures were carried out at the Bionomics Research and Technology Center (BRTC), Rutgers University. The 
microarray labeling, hybridization and analysis procedures were described previously in detail [19]. In brief, each individual sample was subjected to gene expression analysis via the Affymetrix Canine Genome Array, which was subsequently processed and scanned according to the manufacturer's instructions. The GeneChip ${ }^{\circ}$ Canine Genome Array was the first available commercial canine expression array and contains over 23,813 Canis familiaris probe sets to monitor gene expression for over 16,000 Canis familiaris full length transcripts.

\section{Microarray data analysis}

All arrays referred to in this study were assessed for "array performance" prior to data analysis. This process involved the statistical analysis of control transcripts that are spiked into the samples themselves and the hybridization cocktail to assess array performance. In addition, several genes have been identified on each array to help assess the overall quality of signal intensity from all arrays. The results of this analysis were helpful to validate the reproducibility of each array at baseline, allowing us to define the lower level of sensitivity sufficient to identify small changes in biologically relevant genes.

Prior to analysis, data from each hybridization were processed using Microarray Suite software, v 5.0 (MAS 5.0) to yield average difference values corresponding to signal intensity for each probe-set. Distinct algorithms were used to determine the absolute call, which distinguishes the presence or absence of a transcript, the differential change in gene expression (increase (I), decrease (D), marginal increase (MI), marginal decrease (MD), and no change (NC)), and the magnitude of change, which is represented as signal log ratio (on a $\log$ base 2 scale). In brief, the algorithm which defines the presence or absence of a gene takes into consideration several qualitative and quantitative metrics from the raw data set. T-tests were performed on the normalized signal values prior to exploring additional analyses.

All the hybridization data have been submitted to the National Center for Biotechnology Information (NCBI) Gene Expression Omnibus database GEO: http://www. ncbi.nlm.nih.gov/geo with GEO Accession Numbers for series GSE14887. The raw pixels data were imported into GeneTraffic MULTI and all subsequent analyses were performed on a GeneTraffic server (GeneTraffic v. v. 3.2-11, Iobion Informatics, La Jolla, CA). All the microarray data were analyzed and normalized using a Robust Multi-Chip Analysis algorithm (RMA). The average of all the control animal data sets was used as the baseline for the analysis.

\section{Statistical Analysis}

Hemodynamic data were expressed as mean \pm SEM and compared by one-way ANOVA followed by Dunnet's test. For the microarray analysis, statistical significance for changes in gene expression was performed in GeneTraffic using a two-class method ( $t$-test) and with variance stabilization. Due to the overwhelming amount of information generated from microarray analysis, differences were considered statistically significant at a nominal significance of $P \leq 0.01$ and at least \pm 2 -fold change in expression between control and DM dogs. However, for a subset of genes of interest (such as gp91), we considered $P \leq 0.05$ as a significant value.

\section{Results}

\section{Hemodynamics and cardiac function}

The hemodynamic data for these dogs were measured in this study and they were similar to our previously reported data $[11,12,17]$. In our previous studies; we have shown that insulin levels in plasma were significantly decreased, whereas glucose levels in plasma were significantly increased during the development of diabetes (all $P<0.05$ ). In this current study with new group of animals, we also showed that induction of diabetes mellitus increased arterial glucose levels from $74 \pm$ 2 to $291 \pm 32$ at week 1 , to $310 \pm 33$ at week 2 , to $317 \pm$ 41 at week 3 , and to $279 \pm 43$ at week 4 , respectively $(P<0.05$ vs. control) similar to our previous data [17]. Furthermore, dogs had clinical signs of diabetes, i.e., polyuria, polydipsia and weight loss $(25.1 \pm 0.7$ to $20.5 \pm$ $0.9 \mathrm{~kg}$ at week 4).

In addition, our current data showed that LVSP was reduced $(125 \pm 6 \mathrm{mmHg}$ to $107 \pm 8 \mathrm{mmHg})$ and $\mathrm{LV}$ $\mathrm{dP} / \mathrm{d} t$ did not change from control $(2762 \pm 216$ to 2374 $\pm 343 \mathrm{mmHg} / \mathrm{s}$ ) during diabetes. MAP decreased gradually, and this reduction reached statistical significance $102 \pm 7$ to $91 \pm 6 \mathrm{mmHg}$ at week 4. Moreover, at week 4 of diabetes, heart rate and mean CBF were significantly diminished ( $28 \pm 5 \mathrm{ml} / \mathrm{min}$ to $18 \pm 4 \mathrm{ml} / \mathrm{min}$ ) and (100 \pm 7 to $76 \pm 14$ beats $/ \mathrm{min})$, respectively, compared with control $(P<0.05)$.

\section{Global gene transcript profiling}

Our microarray analysis was performed on heart tissues taken at 4 weeks after induction of DM. From our current and previously published data [17], this is the time when glucose uptake was low. Earlier times were mainly fueled by keto-acid uptake. When statistical analysis was performed using $P \leq 0.01$ and \pm 2.0 fold change in comparison to the control group, our result showed that 797 genes were differentially expressed in DM in comparison to control dogs. Of these, 150 genes were expressed at significantly greater levels in diabetic dogs and 647 were significantly reduced. For a full list of differentially expressed genes see additional file 1, Table S1. Unfortunately, about half of the genes in the dog genome remain unidentified, due to the lack of annotation. 
When considering the identified genes, we found that the majority of the genes presented encoded for proteins involved in 104 KEGG (Kyoto Encyclopedia of Genes and Genomes) pathways. The top 12 pathways with the highest number of genes involved are shown in Table 1. We have divided some genes into functional categories and have presented examples of some of them in Table 2. These data showed that a large number of the down regulated genes are involved in metabolic pathways (Table 2). There was down regulation of some of the genes involved in the mitochondrial transport system (Table 2). Furthermore, there was differential down regulation of some of the genes involved in $\mathrm{Ca}^{2+}$ cycling and in myocardial contraction and relaxation (Table 2) in addition to down regulation of genes involved in glutathione pathway (Figure 1). There was up regulation of some components of the NADPH oxidase subunits (gp91 by 2.2 fold, $\mathrm{P}<0.03$ ) and down-regulation of SOD1 (3 fold, $\mathrm{P}<0.001$ ) (Figure 1).

\section{Discussion}

Some of the genes that we found markedly up- or down regulated encode for proteins whose role in myocardial physiology and pathophysiology is well defined. Other genes are presently difficult to put in a pathophysiolgical context. It is obviously impossible to examine in detail functions and potential pathophysiological roles of all of the identified alterations, therefore our discussion will focus on selected genes whose expression was found markedly altered and whose potential importance is supported by the existing literature.

\section{Regulation of cardiac metabolic and structural genes} A large number of the down regulated genes are involved in metabolic pathways. Interestingly, as seen in Table 2, the majority of the genes in the citric acid cycle and the respiratory chain are down regulated by many

Table 1 Shows the top 12 KEEG pathways with the highest number of genes involved

\begin{tabular}{lc}
\hline KEGG Pathway & Number of genes \\
\hline Ribosome & 27 \\
Oxidative phosphorylation & 21 \\
Focal adhesion & 7 \\
Citrate cycle (TCA cycle) & 6 \\
Glutathione metabolism & 6 \\
Glycolysis/Gluconeogenesis & 6 \\
Regulation of actin cytoskeleton & 6 \\
Adherens junction & 5 \\
Calcium signaling pathway & 5 \\
Leukocyte transendothelial migration & 5 \\
PPAR signaling pathway & 5 \\
Wnt signaling pathway & 5 \\
\hline
\end{tabular}

fold decrease. The large down regulation of these genes may be indicative of mitochondrial dysfunction in DM dogs. In our previous studies [12], we have shown that there is depressed modulation of $\mathrm{O}_{2}$ consumption by endogenous $\mathrm{NO}$ in cardiac muscle from diabetic dogs. However, in this recent study based on our gene expression profiling of cardiac tissues from DM dogs, there is strong evidence of a dysfunction of the mitochondria in this DM model. This was also evident by the down regulation of some of the genes involved in the mitochondrial transport system. Examples of these are shown in Table 2. Of particular interest are mitochondrial import receptor subunit TOM22, mitochondrial import stimulation factor L subunit, mitochondrial 2-oxoglutarate/ malate carrier protein, glucose transporter type 1, voltage-dependent anion-selective channel protein 3, malonyl CoA-acyl carrier protein transacylase, translocase of inner mitochondrial membrane 50 homolog. This conclusion supports previous observations by other investigators that there is evidence for mitochondrial dysfunction in the hearts from other models of diabetes [13-15,21]. Savabi [21] showed that there were fewer mitochondria in the hearts from diabetic rats and lower $\mathrm{O}_{2}$ consumption rates in isolated mitochondria than those from normal hearts. Other studies [16] also suggested that there was depressed basal $\mathrm{O}_{2}$ consumption by myocytes suspended in medium, decreased calcium uptake by mitochondria, and reduced mitochondrial membrane potential from diabetic rats. The decrease in calcium uptake by mitochondria is also supported in this study in dogs with DM. Table 2 shows examples of some of the genes involved in $\mathrm{Ca}^{2+}$ cycling and in myocardial contraction and relaxation. During the last decade there was accumulating evidence that alterations of excitation-contraction coupling (EC) may play a critical role in the pathophysiology of myocardial failure. Based on our microarray data (Table 2), a large number of genes involved in these processes are down regulated. The level of mRNA of troponin I, troponin C, tropomyosin, calmodulin, ryanodine receptor type 2 (RyR) and SERCA2 were significantly decreased in these animals. Troponin I has been shown to be very sensitive and specific indicator of damage to the myocardium. Another gene which was down regulated was phospholemman which is a cardiac transmembrane protein. Phospholemman has been shown to regulate ion channel activity [22]. It has been proposed that phospholemman regulates the $\mathrm{Na} / \mathrm{K}$ pump in a manner analogous to regulation of the calcium ATPase SERCA 2a by phospholamban [22]. The net effect of these changes in gene expression might be an overall decrease in both diastolic and systolic ventricular function, which may be an adaptive mechanism to protect the surviving myocardium by reducing its energy expenditure. Linke et al [17] and 
Table 2 Examples of some of the genes which are statistically significant in alloxan induced diabetes in dog hearts

\begin{tabular}{|c|c|c|c|}
\hline Affy Ids & Fold-change & P Values & Gene Title \\
\hline \multicolumn{4}{|c|}{ Metabolic genes } \\
\hline \multicolumn{4}{|l|}{ Up-regulated } \\
\hline 1604393_at & 7.31 & 0.01 & inorganic pyrophosphatase \\
\hline \multicolumn{4}{|c|}{ Down-regulated } \\
\hline 1582358_s_at & -78.79 & 0.004 & glyceraldehyde-3-phosphate dehydrogenase \\
\hline 1586136_x_at & -77.17 & 0.006 & cytochrome c oxidase, subunit Vlb polypeptide 1 \\
\hline 1604455_at & -55.33 & 0.002 & Creatine kinase M-type \\
\hline 1583489_s_at & -54.19 & 0.009 & NADH-ubiquinone oxidoreductase $23 \mathrm{kDa}$ subunit, mitochondrial precursor (Complex I-23KD) \\
\hline 1589664_s_at & -26.35 & 0.002 & ATP synthase, $\mathrm{H}+$ transporting, mitochondrial F0 complex, subunit c, isoform 1 \\
\hline 1603350_x_at & -25.11 & 0.003 & Beta enolase (2-phospho-D-glycerate hydro-lyase) \\
\hline 1603755_at & -24.59 & 0.005 & NADH dehydrogenase (ubiquinone) Fe-S protein 6, 13kDa (NADH-coenzyme Q reductase) \\
\hline 1586831_at & -24.42 & 0.006 & Phosphoglycerate mutase 2 \\
\hline 1583253_at & -22.94 & 0.006 & NADH-ubiquinone oxidoreductase Fe-S protein 7 \\
\hline 1604453_at & -22.63 & 0.003 & isocitrate dehydrogenase 2 (NADP+), mitochondrial \\
\hline 1594799_at & -22.47 & 0.007 & NADH dehydrogenase (ubiquinone) Fe-S protein 3, 30kDa (NADH-coenzyme $\mathrm{Q}$ reductase) \\
\hline 1583615_at & -20.11 & 0.01 & inositol polyphosphate-5-phosphatase, 40kDa \\
\hline 1588757_at & -17.75 & 0.003 & NADH dehydrogenase (ubiquinone) 1 beta subcomplex, 7 \\
\hline 1597725_s_at & -17.63 & 0.006 & 3-hydroxyacyl-CoA dehydrogenase type II \\
\hline 1583148_at & -17.39 & 0.008 & ubiquinol-cytochrome $\mathrm{c}$ reductase core protein I \\
\hline 1583152_at & -17.27 & 0.007 & NADH dehydrogenase (ubiquinone) 1 alpha subcomplex, 8, 19kDa \\
\hline 1583193_s_at & -15.89 & 0.003 & isocitrate dehydrogenase 3 , beta subunit isoform b precursor \\
\hline 1592397_at & -15.24 & 0.009 & succinate-CoA ligase, GDP-forming, alpha subunit \\
\hline 1588432_at & -14.22 & 0.009 & cytochrome c-1 \\
\hline 1588302_at & -13.55 & 0.004 & ubiquinol-cytochrome $\mathrm{c}$ reductase subunit \\
\hline 1583550_s_at & -13.55 & 0.008 & Vacuolar ATP synthase $16 \mathrm{kDa}$ proteolipid subunit \\
\hline 1585498_at & -12.82 & 0.01 & Glucose-6-phosphate isomerase (GPI) \\
\hline 1583430_at & -12.64 & 0.01 & Triosephosphate isomerase (TIM) (Triose-phosphate isomerase) \\
\hline 1585763_at & -12.64 & 0.008 & Ubiquinol-cytochrome c reductase complex $11 \mathrm{kDa}$ protein, mitochondrial precursor \\
\hline 1583348_x_at & -12.55 & 0.006 & cytochrome c oxidase, subunit Vlb polypeptide 1 \\
\hline 1604126_at & -10.63 & 0.007 & enoyl Coenzyme A hydratase, short chain, 1, mitochondrial \\
\hline 1584695_at & -7.84 & 0.003 & succinate dehydrogenase complex, subunit B, iron sulfur (Ip) \\
\hline 1604230_at & -7.73 & 0.006 & NADH dehydrogenase 1 beta subcomplex 4 \\
\hline 1598523_at & -6.02 & 0.01 & Glucose-6-phosphate isomerase (GPI) \\
\hline 1589790_s_at & -6.02 & 0.007 & NADH dehydrogenase (ubiquinone) 1 alpha subcomplex, $6,14 \mathrm{kDa}$ \\
\hline 1592755_s_at & -5.24 & 0.008 & Electron transfer flavoprotein-ubiquinone oxidoreductase \\
\hline 1588377_at & -5.17 & 0.01 & 3-methyl-2-oxobutanoate dehydrogenase kinase \\
\hline 1598623_s_at & -4.79 & 0.005 & phosphofructokinase, muscle \\
\hline 1586218_at & -4.76 & 0.004 & Aldose reductase \\
\hline 1590058_s_at & -4.56 & 0.002 & creatine kinase, mitochondrial 2 \\
\hline 1584362_at & -4.44 & 0.01 & NAD-dependent deacetylase sirtuin-3 \\
\hline 1583716_at & -4.03 & 0.004 & aconitase 2, mitochondrial \\
\hline
\end{tabular}

Mitochondria proteins and transport

\section{Down-regulated}

$\begin{array}{llc}\text { 1583895_x_at } & -71.51 & 0.008 \\ \text { 1593894_at } & -22.78 & 0.01 \\ \text { 1587993_x_at } & -11.47 & 0.008 \\ \text { 1585706_x_at } & -11.39 & 0.008 \\ \text { 1588228_at } & -9.85 & 0.006 \\ \text { 1592554_at } & -9.06 & 0.004 \\ \text { 1586690_at } & -7.89 & 0.01\end{array}$

ribosomal protein L29

Mitochondrial import receptor subunit TOM22 homolog

Inner membrane protein OXA1L, mitochondrial precursor (Oxidase assembly 1-like protein) Inner membrane protein OXA1L, mitochondrial precursor (Oxidase assembly 1-like protein) Mitochondrial 39 S ribosomal protein L23 (L23mt) (MRP-L23) (L23 mitochondrial-related protein) 14-3-3 protein epsilon (14-3-3E) (Mitochondrial import stimulation factor $L$ subunit) (MSF $L$ ) NTF2-related export protein 1 
Table 2 Examples of some of the genes which are statistically significant in alloxan induced diabetes in dog hearts (Continued)

\begin{tabular}{lccl}
\hline 1585137_at & -7.52 & 0.01 & Mitochondrial 2-oxoglutarate/malate carrier protein (OGCP) \\
1586284_at & -7.52 & 0.009 & ATP synthase coupling factor 6, mitochondrial precursor (ATPase subunit F6) \\
1582738_at & -7.26 & 0.01 & FXYD domain containing ion transport regulator 1 (phospholemman) \\
1586934_at & -7.11 & 0.008 & Solute carrier family 2, facilitated glucose transporter, member 1 (Glucose transporter type 1) \\
1592219_s_at & -4.44 & 0.01 & transaldolase 1
\end{tabular}

Calcium uptake and myocardial contraction and relaxation

Up-regulated

\begin{tabular}{|c|c|c|c|}
\hline 1606083_at & 4.44 & 0.007 & protein phosphatase 3 (formerly 2B), catalytic subunit, alpha isoform (calcineurin A alpha) \\
\hline \multicolumn{4}{|c|}{ Down-regulated } \\
\hline 1582664_s_at & -48.50 & 0.004 & troponin I type 3 (cardiac) \\
\hline 1582739_s_at & -36.50 & 0.01 & FXYD domain containing ion transport regulator 1 (phospholemman) \\
\hline 1586044_s_at & -20.82 & 0.008 & actinin, alpha 2 \\
\hline 1585703_s_at & -19.97 & 0.006 & beta-actin///actin, gamma 1 \\
\hline 1582670_s_at & -16.56 & 0.006 & myosin, light chain 2, regulatory, cardiac, slow \\
\hline 1592467_at & -15.03 & 0.01 & Tropomyosin 1 (alpha) \\
\hline 1605421_at & -13.27 & 0.009 & phosphodiesterase 1C, calmodulin-dependent 70kDa \\
\hline 1587427_at & -11.24 & 0.008 & ryanodine receptor 2 (cardiac) \\
\hline 1605889_at & -9.85 & 0.004 & Myosin, light polypeptide 2, regulatory, cardiac, slow \\
\hline 1605583_at & -5.17 & 0.009 & cardiac titin \\
\hline 1585044_x_at & 5.13 & 0005 & myosin, light polypeptide 6, alkali, smooth muscle and non-muscle \\
\hline 1589676_s_at & -5.06 & 0.006 & actin, alpha, cardiac muscle 1 \\
\hline 1583557_at & -4.86 & 0.008 & Adenylyl cyclase-associated protein 1 (CAP 1) \\
\hline 1584317_at & -4.86 & 0.005 & smoothelin isoform c \\
\hline 1602057_s_at & -4.50 & 0.01 & calmodulin 1 \\
\hline 1604456_at & -4.44 & 0.004 & troponin $C$, slow \\
\hline 1592238_s_at & -2.40 & 0.01 & SERCA2 \\
\hline
\end{tabular}

Zhao et al $[11,12]$ showed that in alloxan-induced diabetes in dogs there were no changes in LVSP and LV $\mathrm{dP} / \mathrm{dt}$ at rest. In view of the role of sarco/endoplasic reticulum in the cardiac contraction and relaxation process, the observed reduction in gene expression of sarco/endoplasic reticulum calcium handling proteins may explain the depressed cardiac performance in diabetic dogs. Although our sampling protocol focused on the myocardium, others have also shown similar observations in vascular smooth muscle cells (VSMCs) [23]. Searls et al showed that the previously-reported reduced $\mathrm{Ca}^{2+}$ signaling in VSMCs from diabetic animals is likely to be due to the altered distribution and/or levels of $\mathrm{Ca}^{2}$ ${ }^{+}$regulatory proteins including the $\mathrm{IP}_{3} \mathrm{R} \mathrm{Ca}^{2+}$ channels, RyR and SERCA proteins [23].

\section{Regulation of oxidative stress genes}

A number of studies suggested that superoxide is involved in the endothelial dysfunction in experimental diabetic animals and patients with diabetes because superoxide dismutase and other antioxidants could, at least in part, restore the endothelial dysfunction. We have previously shown that in conscious dogs with DM that: 1) baseline CBF was significantly decreased, 2) endothelium-dependent $(\mathrm{ACh})$ coronary vasodilation were impaired, and 3) reflex cholinergic NO-dependent coronary vasodilation was selectively depressed. The most likely mechanism responsible for the depressed reflex cholinergic NO-dependent coronary vasodilation was the decreased bioactivity of NO from the vascular endothelium. Bradykinin reduced oxygen consumption in normal dog heart in vitro and this was reduced in type I DM [12]. The effect of bradykinin was restored by apocynin, tempol or vitamin $\mathrm{C}$; indicating a role for $\mathrm{NADPH}$ oxidase derived superoxide. Interstingly, there was up regulation of some components of the NADPH oxidase subunits (gp91 by 2.2 fold, $\mathrm{P}<0.03$ ) and downregulation of SOD1 (3 fold, $\mathrm{P}<0.001$ ). Over the years, decreased NO bioavailability has been proposed as one of the determinants of vascular damage in diabetes. Recently, Hamed et al have shown that NO and superoxide dismutase play roles in modulating endothelial progenitor cell function in type 2 diabetes mellitus [24].

Oxidative stress is involved in the pathogenesis of diabetic complications in animal models and in humans [3]. Many authors believe that the liver mitochondrial 


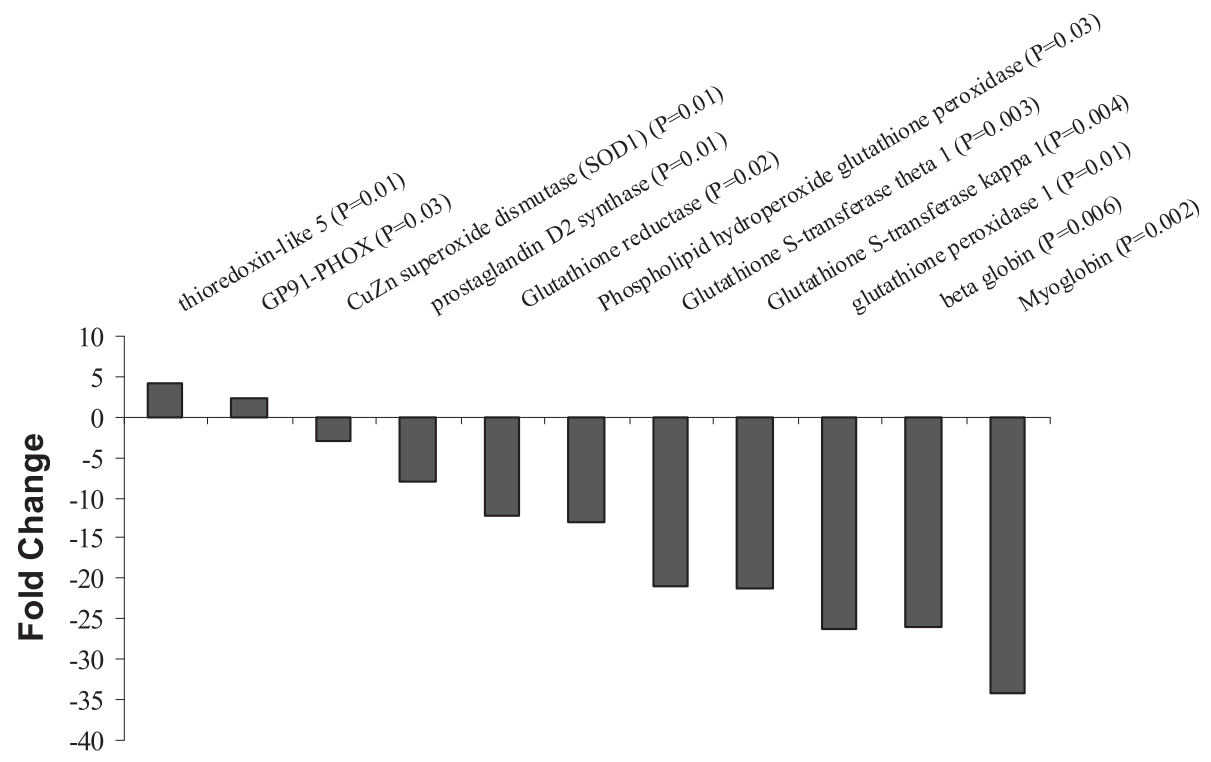

Figure 1 Fold change of some genes involved in the bioactivity of NO that are statistically differentially regulated in the DM dogs. P values are shown for each individual gene.

dysfunction in diabetes is closely dependent on oxidative stress which is enhanced in diabetic animals and patients $[25,26]$. Long-term reactive oxygen species exposure resulted in damage of mitochondrial proteins that caused disturbances in mitochondrial energy production [27]. Some authors suggested that the loss of mitochondrial respiration was essentially due to oxygendependent inactivation of mitochondrial dehydrogenases [28]. The mitochondrial dehydrogenase activities related to complex I (NADH-dehydrogenase) and to complex II (succinate dehydrogenase) were shown to decrease in the diabetic rats. Interestingly, all of the above mentioned genes are also down regulated in our model presented in this study (Table 2).

Oxidative stress may be defined as an imbalance between the production and degradation of reactive oxygen species such as superoxide anion, hydrogen peroxide, lipid peroxides, and peroxynitrite. Enzymatic inactivation of reactive oxygen species is achieved mainly by glutathione peroxidase, superoxide dismutase, and catalase [29]. The peroxidation of membrane lipids is a major consequence of oxidative stress. The oxidation of lipids, particularly phospholipids, has also been suggested to be a significant chemical event in a variety of pathological conditions, such as atherosclerosis, ischemic injury, and carcinogenesis [30]. The formation of lipid hydroperoxides (LOOHs) within the bilayer of membranes disrupts membrane structure and function [31], which subsequently leads to cell injury and death. The repair of intracellular LOOHs can be carried out by cytosolic glutathione peroxidase (cGPx) [32], phospholipid hydroperoxide glutathione peroxidase (PhGPx) [33], nonselenium GPx [34], or glutathione-Stransferase (GST) [35].

In mammalian cells, glutathione and the glutathione peroxidases constitute the principal antioxidant defense system [36]. This is evident by the down regulation of phospholipid hydroperoxide glutathione peroxidase, glutathione peroxidase 1, glutathione reductase (mitochondrial precursor) and glutathione S-transferase. These genes were down regulated by 3.7, 4.7, 3.5 and 2.9 fold respectively (Figure 1 ). There are at least four different glutathione peroxidases, all of which contain selenocysteine at their active sites [37]. Glutathione peroxidase 1, the ubiquitous intracellular form and key antioxidant enzyme within most cells, including the endothelium, uses glutathione to reduce hydrogen peroxide to water and lipid peroxides to their respective alcohols, and it also acts as a peroxynitrite reductase [38]. In mice, glutathione peroxidase 1 deficiency results in abnormal vascular and cardiac function and structure [39]. Recent studies in human have shown that decreased GPX-1 activity was observed in patients with coronary artery disease (CAD) and those with acute myocardial infarction [40]. Espinola-Klein et al [41] have also shown that decreased red blood cell GPX-1 activity is associated with increased cardiovascular risk according to the extent of atherosclerosis.

Glutathione is important in the regulation of the redox state, and a decline in its tissue level has often been considered to be indicative of increased oxidative stress in diabetes. Glutathione (GSH) is present in most 
mammalian cells and plays an important role in cellular defense against oxidative stress by reducing protein disulfides and other cellular molecules. It also acts as a scavenger of free radicals of ROS [42]. GSH is synthesized intracellularly by two GSH-synthesizing enzymes, g-glutamylcysteine synthetase (g-GCS) and glutathione synthetase. g-GCS catalyzes the rate-limiting step of GSH synthesis [43]. In many cells, the GSH redox cycle is catalyzed by both glutathione peroxidase and glutathione reductase. During oxidative stress, the reduced form of GSH is converted by glutathione peroxidase to oxidized glutathione (GSSG). It was reported the presence of low GSH and high GSSG concentrations in erythrocytes of diabetic patients [44] and in endothelial cells of diabetic rabbits [16].

Two of the genes that were highly down-regulated in $\mathrm{DM}$ were Myoglobin $(\mathrm{Mb})$ and beta globin (Figure 1). Previous studies have shown the role of myoglobin in the antioxidant defense of the heart [45]. The lack of myoglobin has been shown to cause a biochemical shift in cardiac substrate utilization from fatty acid to glucose oxidation [46]. In our previous studies [17] we have shown that there was a shift in metabolic uptake by the heart during development of alloxan-induced diabetes at a time when myocardial NO production is already decreased (3-4 weeks). This switch is a well know marker for heart failure. Studies have also suggested that human $\mathrm{Mb}$ actively participates in the regulation of $\mathrm{NO}$ [47]. $\mathrm{Mb}$ is a scavenger of bioactive $\mathrm{NO}$ through the reaction $\mathrm{NO}$ with $\mathrm{MbO}_{2}$ to form metMb and nitrate and may be the major mechanism of attenuating intracellular NO bioactivity in cardiac muscle [45]. Flogel et al showed that hearts from Mb-deficient (myo-/-) mice were found to be more sensitive to the infusion of reactive oxygen species (ROS, e.g., $\mathrm{H}_{2} \mathrm{O}_{2}$ and superoxide) in that depression of myocardial contractile force was more pronounced as compared with WT controls. Furthermore, Mb-deficient hearts released significantly more ROS during IR, and this was accompanied by a delayed functional and metabolic recovery after the ischemic insult. It is obvious in this study that the array data show a direct effect of diabetes on the heart by impairing NO bioavailability through oxidative stress. Therefore, our study is novel in that it represents the first report of cardiac gene expression changes in a canine model of alloxan-induced type 1 diabetes.

\section{Limitations}

Although the analysis of gene expression using oligonucleotide microarrays is a powerful technique, limitations warrant mention. Not all dog genes are represented on the Affymetrix GeneChip ${ }^{\circ}$ Canine Genome Arrays used in this study. Furthermore, the annotations of high number of the genes are still unknown and therefore the knowledge that can be acquired from these experiments remains incomplete. Up to now, this version of the Canine array contains 7,340 complete annotated dog genes. From our previous publications $[19,20,48,49]$ in addition to other reported publication [50], there seems to exist a good correlation between oligonucleotide microarray and qRT-PCR data. This correlation also holds true when ratios of gene expression in different tissues were compared for highly expressed genes and not for those expressed at low levels due to the smaller dynamic range of microarrays [50]. It is well known that alloxan is specifically cytotoxic to the pancreatic $B$ cells. However, the precise mechanism for this selectivity is still not clear. Several studies in alloxan metabolism [51] have documented that $45 \%$ of injected alloxan had been excreted in the urine in 24 hours and $62 \%$ in 3 days. Therefore, the gene expression data from this study where dogs were sacrificed after 4 weeks of alloxan injection are most likely due to the chronic effect of diabetes rather than the acute toxic effect of alloxan.

\section{Conclusion}

The gene expression profiling presented in this study suggests a direct effect of type 1 diabetes on the heart by impairing NO bioavailability through oxidative stress, impairing calcium uptake by the mitochondrial transport system, lipid peroxidases and down regulation of citric acid cycle and the respiratory chain which are indicative of mitochondrial dysfunction in DM dogs. The order of these events is hard to elucidate and is yet to be determined.

\section{Additional material}

Additional file 1: Table S1. Full list of genes with increased and decreased expression in DM versus control dogs.

\section{List of abbreviations}

DM: diabetes mellitus; CBF: coronary blood flow; NO: nitric oxide; eNOS: endothelial nitric oxide synthase; SOD: superoxide dismutase; HF: heart failure; $\mathrm{O}_{2}$ : oxygen; LV: left ventricle; LVSP: LV systolic pressure; HR: heart rate; MAP: mean arterial pressure.

\section{Competing interests}

The authors declare that they have no competing interests.

\section{Authors' contributions}

CO designed the gene array study, performed all the microarray and data analysis and wrote the manuscript. SK Performed the hemodynamics on the animals. FAR participated in editing the manuscript and THH designed the study and revised and approved the final manuscript. All authors have read and approved the manuscript.

\section{Acknowledgements}

This study was supported by the National Heart, Lung, and Blood Institute Grants P01-HL-43023, R01-HL50142, HL61290 (T. H. Hintze) and, in part, by PO1-HL-74237 (F.A. Recchia). Fabio A. Recchia is an Established Investigator of the American Heart Association. 
Received: 19 May 2010 Accepted: 24 August 2010

Published: 24 August 2010

\section{References}

1. Scoppola A, Montecchi FR, Menzinger G, Lala A: Urinary mevalonate excretion rate in type 2 diabetes: role of metabolic control. Atherosclerosis 2001, 156:357-61.

2. Unwin N, Setel P, Rashid S, Mugusi F, Mbanya JC, Kitange H, Hayes L, Edwards R, Aspray T, Alberti KG: Noncommunicable diseases in subSaharan Africa: where do they feature in the health research agenda? Bull World Health Organ 2001, 79:947-953.

3. Caballero AE, Arora S, Saouaf R, Lim SC, Smakowski P, Park JY, King GL, LoGerfo FW, Horton ES, Veves A: Microvascular and macrovascular reactivity is reduced in subjects at risk for type 2 diabetes. Diabetes 1999, 48:1856-1862.

4. Kassab E, McFarlane SI, Sower JR: Vascular complications in diabetes and their prevention. Vasc Med 2001, 6:249-55.

5. Betteridge DJ: Diabetic dyslipidaemia: treatment implications. J Intern Med Supp/ 1994, 736:47-52

6. Regensteiner JG, Sippel J, McFarling ET, Wolfel EE, Hiatt WR: Effects of noninsulin-dependent diabetes on oxygen consumption during treadmill exercise. Med Sci Sports Exerc 1995, 27:875-81.

7. Franssila-Kallunki A, Groop L: Factors associated with basal metabolic rate in patients with type 2 (non-insulin-dependent) diabetes mellitus. Diabetologia 1992, 35:962-6.

8. Avesani CM, Cuppari L, Silva AC, Sigulem DM, Cendoroglo M, Sesso R, Draibe SA: Resting energy expenditure in pre-dialysis diabetic patients. Nephrol Dial Transplant 2001, 16:556-565.

9. Diederich D, Skopec J, Diederich A, Dai FX: Endothelial dysfunction in mesenteric resistance arteries of diabetic rats: role of free radicals. Am J Physiol 1994, 266:H1153-1161.

10. Kiff RJ, Gardiner SM, Compton AM, Bennett T: The effects of endothelin-1 and NG-nitro-L-arginine methyl ester on regional haemodynamics in conscious rats with streptozotocin-induced diabetes mellitus. $\mathrm{Br} J$ Pharmacol 1991, 103:1321-1326.

11. Zhao G, Zhang X, Smith CJ, Xu X, Ochoa M, Greenhouse D, Vogel T, Curran C, Hintze TH: Reduced coronary NO production in conscious dogs after the development of alloxan-induced diabetes. Am J Physiol 1999, 277:H268-278.

12. Zhao G, Zhang $X, X u X$, Wolin MS, Hintze TH: Depressed modulation of oxygen consumption by endogenous nitric oxide in cardiac muscle from diabetic dogs. Am J Physiol Heart Circ Physiol 2000, 279:H520-527.

13. Flarsheim CE, Grupp IL, Matlib MA: Mitochondrial dysfunction accompanies diastolic dysfunction in diabetic rat heart. Am J Physiol Heart Circ Physiol 1996, 271:H192-202.

14. Kuo TH, Moore KH, Giacomelli F, Wiener J: Defective oxidative metabolism of heart mitochondria from genetically diabetic mice. Diabetes 1983, 32:781-787.

15. Mokhtar N, Lavoie J-P, Rousseau-Migneron R, Nadeau A: Physical training reverses defect in mitochondrial energy production in heart of chronically diabetic rats. Diabetes 1993, 42:682-687.

16. Tanaga $\mathrm{Y}$, Konno N, Kako KJ: Mitochondrial dysfunction observed in situ in cardiomyocytes of rats in experimental diabetes. Cardiovasc Res 1992, 26:409-414.

17. Linke A, Zhao G, Recchia FA, Williams J, Xu X, Hintze TH: Shift in metabolic substrate uptake by the heart during development of alloxan-induced diabetes. Am J Physiol Heart Circ Physiol 2003, 285:H1007-1014.

18. Sun G: Application of DNA microarrays in the study of human obesity and type 2 diabetes. OMICS 2007, 11:25-40.

19. Ojaimi C, Qanud K, Hintze TH, Recchia FA: Altered expression of a limited number of genes contributes to cardiac decompensation during chronic ventricular tachypacing in dogs. Physiol Genomics 2007, 29:76-83.

20. Ojaimi C, Li W, Kinugawa S, Post H, Csiszar A, Pacher P, Kaley G, Hintze TH: Transcriptional basis for exercise limitation in male eNOS-knockout mice with age: heart failure and the fetal phenotype. Am J Physiol Heart Circ Physiol 2005, 289:H1399-407.

21. Savabi F: Mitochondrial creatine phosphokinase deficiency in diabetic rat heart. Biochem Biophys Res Commun 1988, 154:469-475.

22. Fuller W, Shattock MJ: Phospholemman and the cardiac sodium pump: protein kinase C, take a bow. Circ Res 2006, 99:1290-1292.
23. Searls YM, Loganathan R, Smirnova IV, Stehno-Bitte L: Intracellular $\mathrm{Ca}^{2+}$ regulating proteins in vascular smooth muscle cells are altered with type 1 diabetes due to the direct effects of hyperglycemia. Cardiovasc Diabetol 2010, 9:8.

24. Hamed S, Brenner B, Aharon A, Daoud D, Roguin A: Nitric oxide and superoxide dismutase modulate endothelial progenitor cell function in type 2 diabetes mellitus. Cardiovasc Diabetol 2009, 8:56.

25. Bakker SJ, IJzerman RG, Teerlink T, Westerhoff HV, Gans RO, Heine RJ: Cytosolic triglycerides and oxidative stress in central obesity: the missing link between excessive atherosclerosis, endothelial dysfunction, and beta-cell failure? Atherosclerosis 2000, 148:17-21.

26. Kristal BS, Koopmans SJ, Jackson CT, Ikeno Y, Park BJ, Yu BP: Oxidantmediated repression of mitochondrial transcription in diabetic rats. Free Radic Biol Med 1997, 22:813-22.

27. Wallace DC: Mitochondrial diseases in man and mouse. Science 1999, 283:1482-1488

28. Aggarwal BB, Quintanilha AT, Cammack R, Packer L: Damage to mitochondrial electron transport and energy coupling by visible light. Biochim Biophys Acta 1978, 502:367-382.

29. Forsberg $L$, de Faire $U$, Morgenstern R: Oxidative stress, human genetic variation, and disease. Arch Biochem Biophys 2001, 389:84-93.

30. Berliner A, Heinecke JW: The role of oxidized lipoproteins in atherogenesis. Free Radic Biol Med 1996, 20:707-727.

31. Pradhan D, Weiser M, Lumley-Sapanski K, Frazier D, Kemper S, Williamson P, Schlegel RA: Peroxidation-induced perturbations of erythrocyte lipid organization. Biochim Biophys Acta 1990, 1023:398-404.

32. Sevanian A, Mukkassah-Kelley SF, Montestruque S: The influence of phospholipase $\mathrm{A} 2$ and glutathione peroxidase on the elimination of membrane lipid peroxides. Biochim Biophys Acta 1983, 223:441-452

33. Thomas JP, Ursini MF, Girotti AW: Protective action of phospholipid hydroperoxide glutathione peroxidase against membrane-damaging lipid peroxidation. In situ reduction of phospholipid and cholesterol hydroperoxides. J Biol Chem 1990, 265:454-461.

34. Fisher AB, Dodia C, Manevich Y, Chen JW, Feinstein SI: Phospholipid hydroperoxides are substrates for non-selenium glutathione peroxidase. J Biol Chem 1999, 274:21326-21334

35. Hurst R, Bao Y, Jemth P, Mannervik B, Williamson G: Phospholipid hydroperoxide glutathione peroxidase activity of human glutathione transferases. Biochem J 1998, 332:97-100.

36. Raes M, Michiels C, Remacle J: Comparative study of the enzymatic defense systems against oxygen-derived free radicals: the key role of glutathione peroxidase. Free Radic Biol Med 1987, 3:3-7.

37. Arthur JR: The glutathione peroxidases. Cell Mol Life Sci 2000 57:1825-1835.

38. Sies $\mathrm{H}$ : Glutathione and its role in cellular functions. Free Radic Biol Med 1999, 27:916-921.

39. Forgione MA, Cap A, Liao R, Moldovan NI, Eberhardt RT, Lim CC, Jones J, Goldschmidt-Clermont PJ, Loscalzo J: Heterozygous cellular glutathione peroxidase deficiency in the mouse: abnormalities in vascular and cardiac function and structure. Circulation 2002, 106:1154-1158.

40. Loeper J, Goy L, Rozensztazn O Bedu, Moisson P: Lipid peroxidation and protective enzymes during myocardial infarction. Clin Chim Acta 1991, 196:119-125

41. Espinola-Klein C, Rupprecht HJ, Bickel C, Schnabel R, Genth-Zotz S, Torzewski M, Lackner K, Munzel T, Blankenberg S, AtheroGene Investigators: Glutathione peroxidase-1 activity, atherosclerotic burden, and cardiovascular prognosis. Am J Cardiol 2007, 99:808-12.

42. Meister A: Methods for the selective modification of glutathione metabolism and study of glutathione transport. Methods Enzymol 1985, 113:571-585

43. Richmann PG, Meister A: Regulation of g-glutamyl-cysteine synthetase by nonallosteric feedback inhibition by glutathione. J Biol Chem 1975, 250:1422-1426.

44. Murakami K, Kondo T, Otsuka Y, Fujiwara Y, Shimada M, Kawakami Y: Impairment of glutathione metabolism in erythrocytes from patients with diabetes mellitus. Metabolism 1989, 38:753-758

45. Flögel U, Gödecke A, Klotz LO, Schrader J: Role of myoglobin in the antioxidant defense of the heart. FASEB J 2004, 18:1156-8.

46. Flögel U, Laussmann $T$, Gödecke $A$, Abanador N, Schäfers $M$, Fingas $C D$, Metzger S, Levkau B, Jacoby C, Schrader J: Lack of myoglobin causes a switch in cardiac substrate selection. Circ Res 2005, 96:e68-75. 
47. Li W, Jue T, Edwards J, Wang X, Hintze TH: Changes in NO bioavailability regulate cardiac $\mathrm{O} 2$ consumption: control by intramitochondrial SOD2 and intracellular myoglobin. Am J Physiol Heart Circ Physiol 2004, 286 H47-54.

48. Suematsu N, Ojaimi C, Kinugawa S, Wang Z, Xu X, Koller A, Recchia FA, Hintze TH: Hyperhomocysteinemia alters cardiac substrate metabolism by impairing nitric oxide bioavailability through oxidative stress. Circulation 2007, 115:255-262.

49. Williams JG, Ojaimi C, Qanud K, Zhang S, Xu X, Recchia FA, Hintze TH: Coronary nitric oxide production controls cardiac substrate metabolism during pregnancy in the dog. Am J Physiol Heart Circ Physiol 2008, 294: H2516-2523.

50. Czechowski T, Bari RP, Stitt M, Scheible WR, Udvardi MK: Real-time RT-PCR profiling of over 1400 Arabidopsis transcription factors: unprecedented sensitivity reveals novel root- and shoot-specific genes. Plant J 2004, 38:366-379.

51. Lee JM, Stetten D Jr: Studies in alloxan metabolism. I. The distribution and excretion of injected alloxan. J Biol Chem 1952, 197:205-214.

doi:10.1186/1475-2840-9-43

Cite this article as: Ojaimi et al:: Oxidant-NO dependent gene regulation in dogs with type I diabetes: impact on cardiac function and metabolism. Cardiovascular Diabetology 2010 9:43.

\section{Submit your next manuscript to BioMed Central} and take full advantage of:

- Convenient online submission

- Thorough peer review

- No space constraints or color figure charges

- Immediate publication on acceptance

- Inclusion in PubMed, CAS, Scopus and Google Scholar

- Research which is freely available for redistribution

Submit your manuscript at www.biomedcentral.com/submit 